\title{
INSULATOR-TO-METAL TRANSITION IN POLYANILINE
}

\section{A.J. EPSTEIN}

Department of Physics and Department of Chemistry, The Ohio State University, Columbus, $\mathrm{OH} 43210$ (U.S.A.)

J.M. GINDER and F. ZUO

Department of Physics, The Ohio State University, Columbus, OH 43210 (U.S.A.)

R.W. BIGELOW

Xerox Webster Research Center, Webster, NY 14580 (U.S.A.)

H.-S. WOO and D.B. TANNER

Department of Physics, University of Florida, Gainesville, FL 32611 (U.S.A.)

A.F. RICHTER, W.-S. HUANG and A.G. MacDIARMID

Department of Chemistry, University of Pennsylvania, PA 19104 (U.S.A.)

\begin{abstract}
The emeraldine base (EB) form of polyaniline can be varied from insulating $\left(\sigma<10^{-10} \mathrm{ohm}^{-1} \mathrm{~cm}^{-1}\right)$ to conducting $\left(\sigma \approx 10^{+1} \mathrm{ohm}^{-1} \mathrm{~cm}^{-1}\right)$ through protonation. That is, the number of electrons on the polymer backbone is constant while the number of protons is increased. We present here extensive magnetic, optical and transport data that demonstrate that the resulting emeraldine salt (ES) is metallic with a ifinite density of states at the Fermi energy. The results are consistent with segregation into fully protonated emeraldine salt and unprotonated emeraldine base polymer regions. It is proposed that the observed transition is an isolated bipolaron-to-polaron lattice transition. The correspondence of this concept to the disproportionation between protonated imine plus amine to form two semiquinones is shown.
\end{abstract}

\section{INTRODUCTION}

Conducting polymers have been under intensive study since the first reported $p$ (oxidative) and $n$ (reductive) doping of polyacetylene [1] and the experimental verification of the onset of a metallic density of states at high doping [2-4]. Polyaniline 
has been described in many papers $[5,6]$ during the past 100 years. The EB form of the polymer is proposed [6] to have equal numbers of reduced $\left[-\left(\mathrm{C}_{6} \mathrm{H}_{4}\right)-\left(\mathrm{N}(\mathrm{H})-\left(\mathrm{C}_{6} \mathrm{H}_{4}\right)-(\mathrm{N}(\mathrm{H})-]\right.\right.$ and oxidized $\left[-\left(\mathrm{C}_{6} \mathrm{H}_{4}\right)-\mathrm{N}=\left(\mathrm{C}_{6} \mathrm{H}_{4}\right)=\mathrm{N}\right.$-] repeat units, Figure 1a. Upon treatment of EB with acids of varying $\mathrm{pH}$, protons, $\mathrm{H}^{+}$, are added to a fraction of the formerly unprotonated nitrogen sites[6,7]. For example, treatment of $\mathrm{EB}$ with aqueous $\mathrm{HCl}$ of $\mathrm{pH}=0.0$ yields nearly complete protonation, i.e., the conducting emeraldine salt, Figure $1 \mathrm{~b}$. Thus, in contrast to polyacetylene, the number of electrons on the polymer backbone is held constant while the number of protons is varied. The protonation reaction is reversable. The conducting form of polyaniline can also be synthesized through the electrochemical oxidation of the fully protonated leucoemeraldine form of polyaniline, Figure 1c $[7,8]$. The determination of the proper description of the resulting conducting state and the establishment of the mechanism for the apparent insulator-to-metal" transition is of central importance especially with respect to the understanding of nonlinear phenomena in polymers with differing repeat units (the "-A = B-" polymer [9]) and nondegenerate ground states [10-12].

(a)

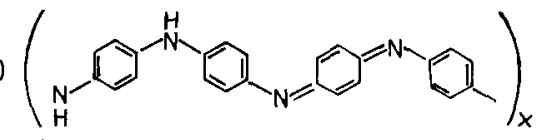

(b)

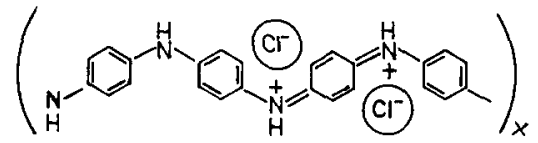

(c)

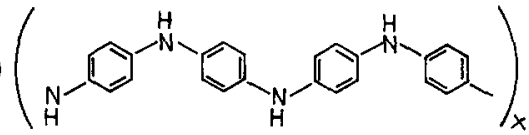

Fig. 1. (a) Emeraldine base, (b) emeraldine salt, and (c) leucoemeraldine base.

\section{EXPERIMENTAL TECHNIQUES}

The chemical preparation of the emeraldine base form of polyaniline and its protonation to the salt form is described elsewhere [6,7]. The magnetic susceptibility was measured via a Faraday technique [3] and electron spin resonance. The reflectance for emeraldine was measured with a Michelson interferometer in the far infrared and a grating monochrometer in the infrared and visible [13]; the frequencydependent conductivity was determined by Kramers-Kronig analysis. The optical absorption spectrum of emeraldine as a function of protonation and leucoemeraldine as a function of electrochemical oxidation were obtained using a Beckman DU-8 UVvisible spectrophotometer. The temperature dependent dc conductivity and its electric field dependence were measured together with the thermoelectric power in a previously described apparatus $[4,14]$. 


\section{EXPERIMENTAL RESULTS}

The temperature dependent magnetic susceptibility, $X^{\exp }$, of selected samples of emeraldine as a function of protonation (chlorine to nitrogen ratio) is shown in Fig. 2. Decomposing the experimental data into a sum of a temperature dependent term, $\mathrm{x}$ Curie $=\mathrm{n}(\mathrm{T}) \mathrm{C} / \mathrm{T}$ where $\mathrm{C}$ is the Curie constant for a noninteracting spin $\frac{1}{2}$ with $\mathrm{g}=2$, and the temperature independent term, $X^{\text {Core }}+X^{\text {Pauli }}$, and assuming $x^{\text {Pauli }}=0$ for emeraldine base $(\mathrm{EB})$ form, we obtain $\mathrm{X}_{\mathrm{EB}}$ Core $=-106 \times 10^{-6} \mathrm{emu} / \mathrm{mole}-2 \mathrm{ring}$. This value is consistent with the proposed formulation Fig. 1a. In addition, approximately one Curie spin per 400 rings is observed.

Using this core value together with $\mathrm{X}_{\mathrm{Cl}}$ Core $=-24 \times 10^{-6} \mathrm{emu} / \mathrm{mole}-2$ ring, we obtain $x^{\text {Pauli }}$ as a function of protonation, Fig. 3. For highly protonated samples, $x$ Pauli $=100 \times 10^{-6} .(\mathrm{emu} / \mathrm{mole} 2 \mathrm{rings})=7.1 \times 10^{-6}(\mathrm{emu} / \mathrm{mole}(\mathrm{C}+\mathrm{N})[15]$ as compared with $3 \times 10^{-6} \mathrm{emu} / \mathrm{mole} \mathrm{C}$ for polyacetylene at $\sim .06$ dopant per carbon [3]. In contrast to the doping of polyacetylene, both the Pauli susceptibility and the number of Curie like spins increase with protonation.

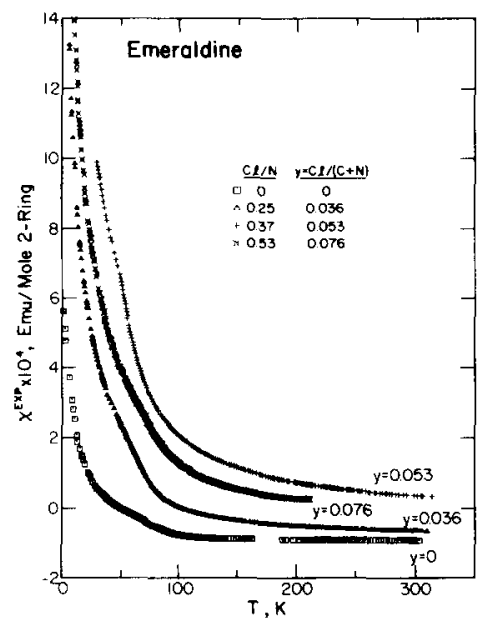

Fig. 2. Representative $x^{\exp }$ versus $T$.

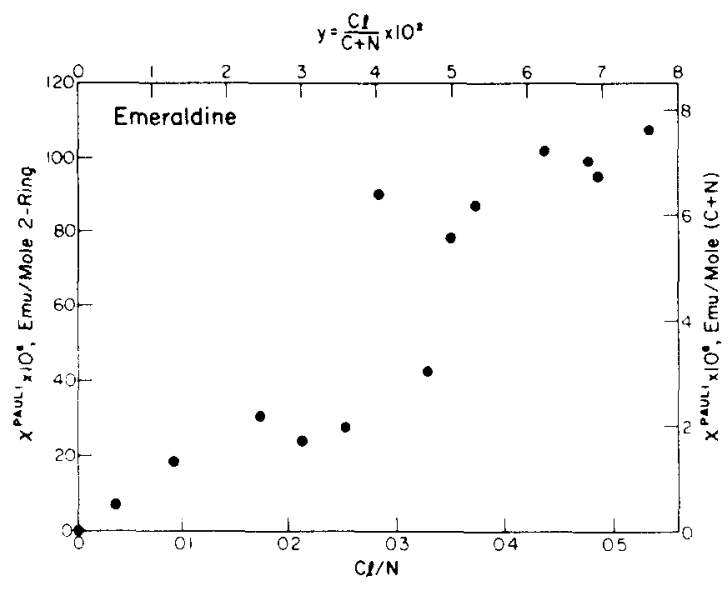

Fig. 3. xPauli vs. Cl/N ratio for emeraldine.

The electronic structure changes substantially upon protonation. Figure 4a compares the optical absorption of the EB (unprotonated, solid line) and ES (dashed line) forms. Upon protonation, the bandgap absorption remains at $\hbar \omega \geqslant 3.5 \mathrm{eV}$ while the $2.1 \mathrm{eV}$ ingap absorption disappears and two new ingap absorptions at $\sim 1.5 \mathrm{eV}$ and $\sim 2.9$ $\mathrm{eV}$ appear. The Kramers-Kronig analysis of reflectance data for the protonated samples, (Fig. 4b), is in agreement with this change. In addition, a series of moderately strong infrared modes are observed, Fig. 4c. The dc conductivity for the emeraldine salt material is plotted in Figure 5. The room temperature conductivity is $\sim 1 \mathrm{ohm} \mathrm{m}^{-1} \mathrm{~cm}^{-1}$ while the thermoelectric power is quasi-metallic. 

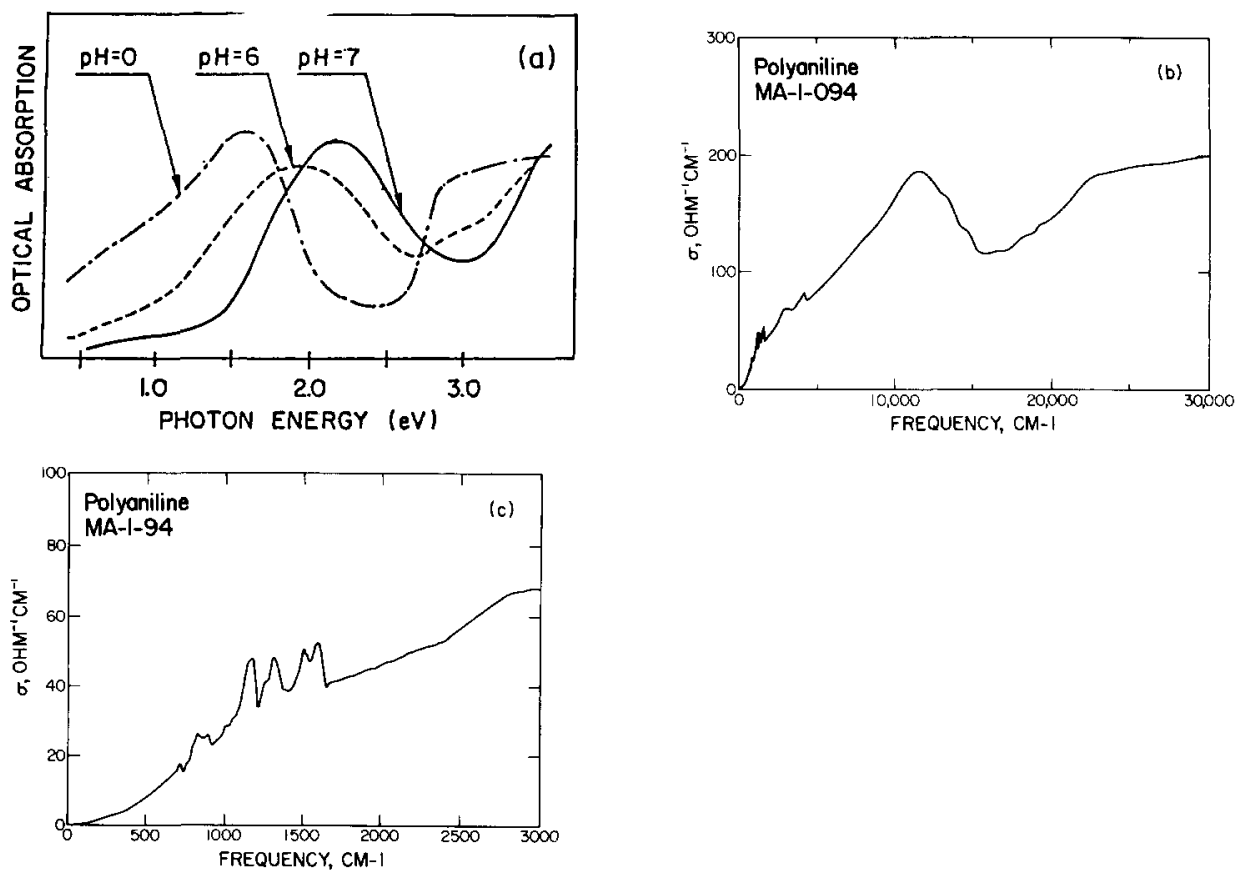

Fig. 4. (a) Optical absorption vs energy: $\mathrm{EB}(\mathrm{pH}=7), \mathrm{ES}(\mathrm{pH}=0)$ and intermediate protonation, (b) and (c) conductivity vs. frequency for ES.

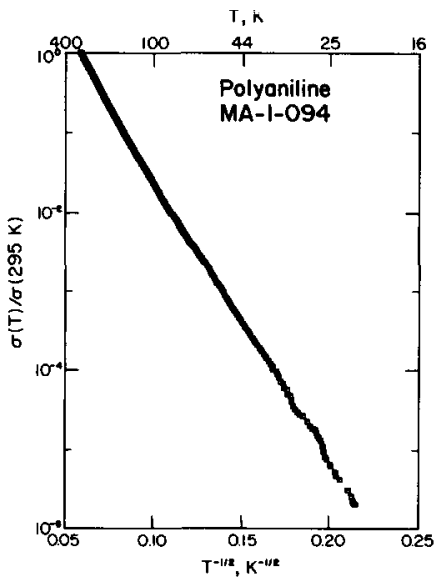

Fig. 5. Log $[\sigma / \sigma(295 \mathrm{~K})]$ vs $\mathrm{T}^{-\frac{1}{2}}$ for $\mathrm{ES}$.

\section{DISCUSSION}

The nearly linear increase in $X^{P a u l i}\left(\mu_{B} 2 N\left(E_{F}\right)\right.$ where $N\left(E_{F}\right)$ is the density of states at the Fermi energy) with percent protonation strongly supports that there is phase segregation into metallic and nonmetallic regions, with $\mathrm{x}^{\text {Pauli }}$ for the metallic regions 
equal to $\sim 110 \times 10^{-6} \mathrm{emu} / \mathrm{mole} \mathrm{H}^{+}$. The phase segregation occurs at chlorine to nitrogen levels as low as 0.1 , and is consistent with the concentration dependence of the room temperature conductivity (as expected for a system dominated by percolation). The temperature dependence is best fit by $\sigma \propto \exp \left(-\left(\mathrm{T}_{0} / \mathrm{T}\right)^{\frac{1}{2}}\right)$ with $\mathrm{T}_{0}=6400 \mathrm{~K}$ for heavily protonated samples, in agreement with the resistivity of granular metals in the concentration regime in which isolated metallic particles are dispersed in a dielectric continuum[21].

The origin of the Pauli susceptibility and hence the finite density of states at the Fermi energy, $N\left(E_{F}\right)=X^{P a u l i} / \mu_{B} 2$ is important in the context of nondegenerate "generalized" $-\mathrm{A}=\mathrm{B}$ - polymers. The maximum in the optical conductivity at $1.5 \mathrm{eV}$ argues against a metallic state resulting from the overlap between a bipolaron band and the valence band. The high Pauli susceptibility and density of states $(\sim 0.23$ states $/ \mathrm{eV} /(\mathrm{C}+\mathrm{N})$ ) argues against phase disordering of the Peierls semiconductor $[13,14,17]$. The observed spin density is much less than the number of protons added. Formation of isolated bipolarons or an ordered bipolaron lattice (centered on the quinoid rings, Fig. $1 \mathrm{~b}$ and $6 \mathrm{a}$ )-is not consistent with the observed Pauli susceptibility. We propose here that the localized bipolarons (Fig. 6a) undergo a phase transition to an ordered polaron lattice (Fig. 6c). The intermediate virtual state of two polarons centered on adjacent N's (Fig. 6b) is included for clarity. This proposal is supported by the known [18] instability of a diamine and diprotonated diimine towards disproportionation and formation of two semiquinone positive ions. It has some similarity to the soliton lattice to polaron lattice transition model proposed [19] for $n$ doped polyacetylene. The phase segregation into metallic and nonmetallic regions is in accord with the predicted [24] first order nature of such a transition.

The optical spectrum, Figure 4, demonstrates the disappearance of one band in the gap (at $2 \mathrm{eV}$ ) and the formation of two new ones as expected for the disappearance of the localized quinoid structure (exciton absorption) of $\mathrm{EB}$ and the formation of a new

(a)

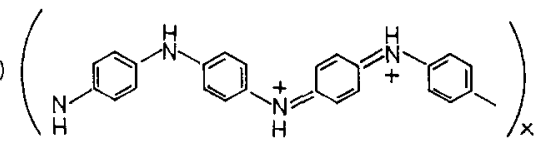

(b)

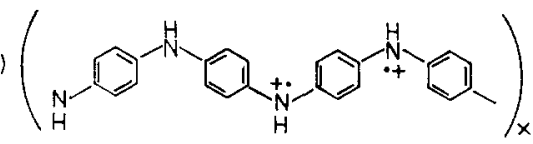

(c)<smiles>[Y]c1ccc(N[C+]=[NH+]c2ccc(Nc3ccc(Nc4ccc(N)cc4)cc3)cc2)cc1</smiles>

Fig. 6. (a) ES as bipolaron $(+2 \mathrm{e})$ in $\mathrm{LE}$, (b) Disassociation of bipolaron $(+2 \mathrm{e})$ to two polarons $(+\mathrm{e})$, (c) Polaron lattice. 
polaron lattice. The relatively small changes in the infrared modes upon protonation are in qualitative agreement with the predictions for a polaron lattice [21].

Assuming a one-dimensional tight-binding model for the polaron band, $\mathrm{X}^{\text {Pauli }}$ yields a polaron band width of $W_{p}=0.37 \mathrm{eV}$. Using the results from continuum theory (including the assumption of a nondegeneration ground state)[19], $\mathrm{W}_{\mathrm{p}}=4 \sqrt{ } 2 \Delta_{\mathrm{o}} \exp [-$ $\left.\mathrm{R} /\left(\sqrt{ } 2 \xi_{0}\right)\right]$ where $\Delta_{0}$ is the half gap $(\sim 2 \mathrm{eV}), \mathrm{R}$ the polaron-polaron spearation, and $\xi_{0}$ the polaron decay length. If $R$ is twice the nitrogen-nitrogen distance $(N-N)$, then $\xi_{0}=0.4 \mathrm{~N}-\mathrm{N}$. Given the wave functions for polarons [12], the charge is expected to spread out considerably along a polyaniline chain.

\section{SUMMARY}

The protonation of the emeraldine base form of polyaniline results in the transition to a metallic state. Extensive studies support that this is a transition from isolated bipolarons to a segregated polaronic metal phase.

\section{ACKNOWLEDGEMENT}

Supported in part by NSF-DMR 841654, DMR-8216718 and Office of Naval Research.

\section{REFERENCES}

1 H. Shirakawa, E.J. Louis, A.G. MacDiarmid, C.K. Chiang and A.J. Heeger, J. C.S.,Chem Comm., (1977) 578; C.K. Chiang, A.G. MacDiarmid, Y.W. Park, and H. Shirakawa, J. Am. Chem. S oc., 100 (1978) 1013.

2 S. Ikehata, J. Kaufer, T. Woerner, A. Pron, M.A. Druy, A. Sivak, A.J. Heeger and A.G. MacDiarmid, Phys. Rev. Lett.,45 (1980) 1123.

3 A.J. Epstein, H. Rommelmann, M.A. Druy, A.J. Heeger and A.G. MacDiarmid, Solid State Commun., 38 (1981) 683.

4 A.J. Epstein, R.W. Bigelow, H. Rommelmann, H.W. Gibson, R.J. Weagley, A. Feldblum, D.B. Tanner, J.P. Pouget, J.C. Pouxviel, R. Comes, P. Robin and S. Kivelson, Mol. Cryst. Liq. Cryst., 117 (1985) 147.

5 A.G. Green and A.E. Woodhead, J. Chem. Soc. (1910) 2388.

6 J.C. Chiang and A.G. MacDiarmid, Synth. Met., 13 (1986) 193 and references therein.

7 A.G. MacDiarmid, J.C. Chiang, W.S. Huang, A.F. Richter and A.J. Epstein, these Proceedings.

8 W.R. Salaneck, I. Lundström, W.S. Huang and A.G. MacDiarmid, Synth. Met., $\underline{13}$ (1986) 291.

$9 \quad$ M.J. Rice and E.J. Mele, Phys. Rev. Lett., 49 (1982) 1455.

10 W.P.Su and J.R. Schrieffer, Proc. Nat. Acad. Sci. U.S.A., 77 (1980) 5526.

11 K. Fesser, A.R. Bishop and D.K. Campbell, Phys. Rev. B, 27 (1983) 4804.

12 Y. Onodera, Phys. Rev. B, 30 (1984) 775. 
13 X.Q. Yang, D.B. Tanner, A. Feldblum, H.W. Gibson, M.J. Rice and A.J. Epstein, Mol. Cryst. Liq. Cryst., 117 (1985) 267.

14 A.J. Epstein, H. Rommelmann, R. Bigelow, H.W. Gibson, D.M. Hoffman and D.B. Tanner, Phys. Rev. Lett., 50 (1983) 1866.

15 J.M. Ginder, A.J. Epstein, R.W. Bigelow, A.F. Richter and A.G. MacDiarmid, Bull. Am. Phys. Soc., 31 (1986) 582.

16 B. Abeles, P. Sheng, M.D. Coutts and Y. Arie, Adv. Phys., 24 (1975) 407.

17 E.J. Mele and M.J. Rice, Phys. Rev. B, 23 (1981) 5397.

18 H. Linschitz, J. Rennert and T.M. Korn, J. Am. Chem. Soc., 76 (1954) 5839; G. Wnek, Polymer Prepr., 27 (1986) 277.

19 S. Kivelson and A.J. Heeger, Phys. Rev. Lett., 55 (1985) 308.

20 B. Horovitz, Phys. Rev. Lett., 55 (1985) 1429.

21 H.Y. Choi and E.J. Mele, submitted for publication. 